\title{
Floating Microplastics in the South Adriatic Sea
}

\author{
G. Suaria ${ }^{1}$, C.G. Avio $^{2}$, G. Lattin ${ }^{3}$, F. Regoli ${ }^{2}$ and S. Aliani ${ }^{1}$ \\ ${ }^{1}$ CNR-ISMAR, La Spezia, Italy ${ }^{2}$ Università Politecnica delle Marche, Ancona, Italy ${ }^{3}$ Algalita Marine \\ Research and Education, Long Beach, CA, United States
}

Neustonic microplastic abundance and polymeric composition were determined after a cruise conducted in the Southern Adriatic Sea between May 9 and 17, 2013. Plankton samples were collected using a neuston net $(200 \mu \mathrm{m}$ mesh size) towed at $\sim 2 \mathrm{kts}$ for 5-6 minutes. In the laboratory, plastic particles were hand-picked using a dissecting stereomicroscope, counted, weighed, and assigned to seven different size classes. Because of the high risk of contamination synthetic fibers were removed from our dataset and not considered in density calculations. On a subset of collected particles $>700 \mu \mathrm{m}(n=869)$, FT-IR analyses were performed to characterize their polymeric

Plastic was found in all samples $(n=30)$. A total of 5163 particles were collected during the survey, the vast majority of which were irregular hard plastic fragments $(97.4 \%)$.

Plastic abundance markedly increased with decreasing size indicating very high fragmentation rates (i.e., $29.4 \%$ of all particles were $<300 \mu \mathrm{m}$ and $53.8 \%$ were $<500 \mu \mathrm{m})$. Overall, an average concentration of $0.83 \pm 1.05$ particles $/ \mathrm{m}^{2}$ and $485.07 \pm 1153.07 \mathrm{~g} / \mathrm{km}^{2}$ was observed throughout the study area, with plastic abundances ranging from 0.04 particles $/ \mathrm{m}^{2}$ to a maximum of 4.65 particles $/ \mathrm{m}^{2}$. 


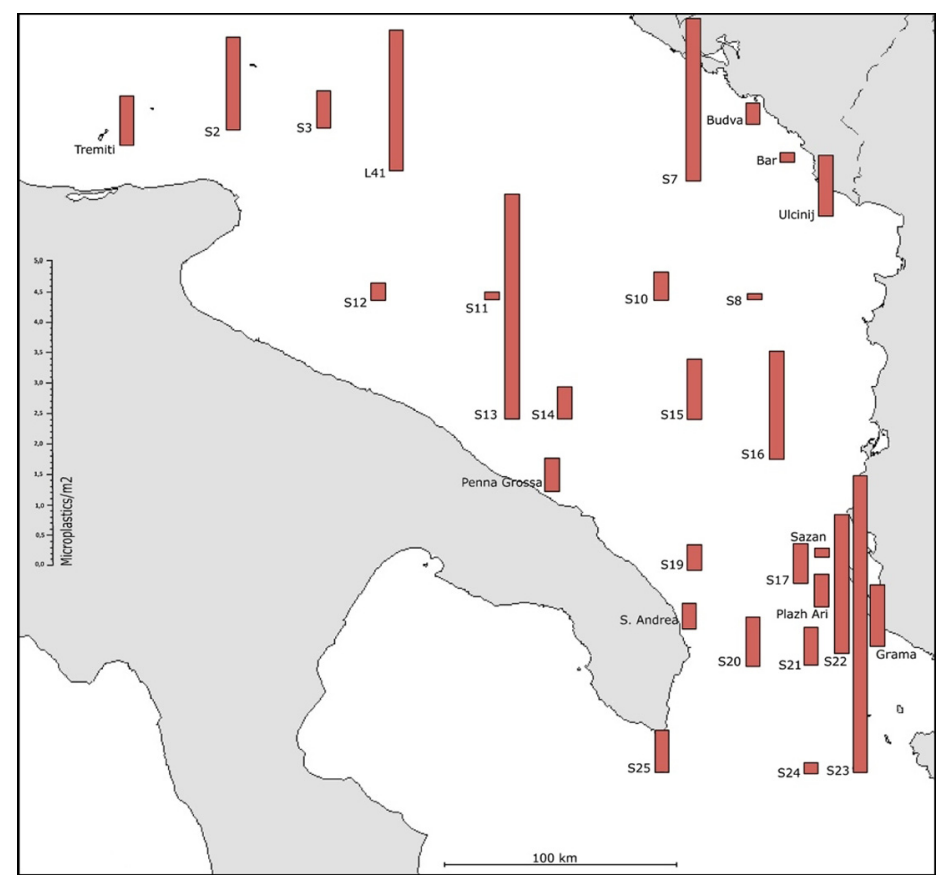

Map of the study area showing the location of all sampling stations and measured microplastic concentrations expressed as number of items $/ \mathrm{m}^{2}$.

Sixteen different polymer classes were identified through FT-IR analyses.

Polyethylene was the predominant polymer $(41.2 \%)$, followed by synthetic paints $(10.6 \%)$, polypropylene $(9.7 \%)$, polyamides $(8.4 \%)$, PVC $(8.1 \%)$, polystyrene $(4.8 \%)$ and polyvinyl-alcohol $(2 \%)$. Other polymers encountered less frequently included PET, cellulose acetate, polyisoprene, poly(vinyl stearate), ethylene-vinyl acetate, epoxy resin, paraffin wax, and 11 fragments of polycaprolactone, a biodegradable polyester.

On the whole, very high levels of plastic pollution were found in the study area. Despite any clear geographical pattern was identified, the conspicuous heterogeneity in plastic distribution and polymeric composition seem to confirm the existence of multiple pollution sources insisting on the Adriatic Sea. 